\title{
Characterization of an inconsistency ranking for pairwise comparison matrices
}

\author{
László Csató* \\ Institute for Computer Science and Control, Hungarian Academy of Sciences (MTA SZTAKI) \\ Laboratory on Engineering and Management Intelligence, Research Group of Operations \\ Research and Decision Systems \\ Corvinus University of Budapest (BCE) \\ Department of Operations Research and Actuarial Sciences \\ Budapest, Hungary
}

June 13, 2017

\begin{abstract}
Pairwise comparisons between alternatives are a well-known method for measuring preferences of a decision-maker. Since these often do not exhibit consistency, a number of inconsistency indices has been introduced in order to measure the deviation from this ideal case. We axiomatically characterize the inconsistency ranking induced by the Koczkodaj inconsistency index: six independent properties are presented such that they determine a unique linear order on the set of all pairwise comparison matrices.
\end{abstract}

Keywords: Pairwise comparisons; Analytic Hierarchy Process (AHP); inconsistency index; axiomatic approach; characterization

JEL classification number: $\mathrm{C} 44$

MSC classes: 90B50, 91B08

\section{Introduction}

Pairwise comparisons are a fundamental tool in many decision-analysis methods such as the Analytic Hierarchy Process (AHP) (Saaty, 1980). However, when different entities ${ }^{1}$ are compared with regard to abstract, non-measurable criteria by fallible humans, it may happen that the set of comparisons is not consistent: for example, entity $A$ is two times better than entity $B$, entity $B$ is three times better than entity $C$, but entity $A$ is not six $(=2 \times 3)$ times better than entity $C$. Inconsistency can also be an inherent feature of the data (see e.g. Bozóki et al. (2016)).

\footnotetext{
* e-mail: laszlo.csato@uni-corvinus.hu

${ }^{1}$ Throughout paper, the term entity is used for the 'things' that are compared. They are sometimes called alternatives, objects, etc.
} 
Therefore, measuring the inconsistency of pairwise comparison matrices - that is, assigning a numerical value to the question 'How much a given matrix deviates from one representing consistent preferences?' - emerges as an important issue. According to our knowledge, the first concept of inconsistency was presented by Kendall and Smith (1940). Since then, a large number of inconsistency indices has been proposed such as Saaty's eigenvalue-based index (Saaty, 1977), the Koczkodaj inconsistency index (Koczkodaj, 1993; Duszak and Koczkodaj, 1994), the relative error (Barzilai, 1998), the geometric consistency index (Aguaron and Moreno-Jiménez, 2003), the inconsistency index by Peláez and Lamata (Peláez and Lamata, 2003), or the harmonic consistency index (Stein and Mizzi, 2007).

Deeper understanding of inconsistency indices is not only a mathematical exercise. Brunelli et al. (2013) and Brunelli (2016a) show that the application of various indices may lead to different, even almost opposite conclusions, so choosing among them properly really counts. It is not by chance that a number of articles deals with the study and comparison of different inconsistency indices (see e.g. Bozóki and Rapcsák (2008) and Brunelli et al. (2013)). Recently, an axiomatic approach has been followed: the introduction and justification of reasonable properties may help to narrow the general definition of inconsistency index as well as highlight those problematic indices that do not satisfy certain requirements (Brunelli and Fedrizzi, 2011; Brunelli, 2016a, 2017; Brunelli and Fedrizzi, 2015; Cavallo and D'Apuzzo, 2012; Koczkodaj and Szwarc, 2014; Koczkodaj and Szybowski, 2015).

Nonetheless, while logical consistency (Is the set of properties devoid of logical contradiction? Does there exist any inconsistency index that satisfies all axioms?) and independence (Is there any redundant axiom, implied by some of the others?) are usually discussed by the authors (Brunelli, 2017; Brunelli and Fedrizzi, 2015), we do not know any result on the axiomatic characterization of inconsistency indices.

Characterization means proposing a set of properties such that there exists a unique measure satisfying all requirements. It is an extensively used approach in social choice, illustrated by the huge literature, for instance, on the axiomatization of the Shapley value in game theory (see e.g. Shapley (1953); Dubey (1975); Young (1985); Hart and Mas-Colell (1989); van den Brink (2002)), or on the Hirsch index in scientometrics (see e.g. Woeginger (2008); Quesada (2010); Miroiu (2013); Bouyssou and Marchant (2014)). It is not unknown in the case of methods used for deriving weights from pairwise comparison matrices (Fichtner, 1984, 1986; Barzilai, 1997), too.

The central contribution of this study is a characterization of the so-called Koczkodaj inconsistency ranking, induced by the Koczkodaj inconsistency index. The first axiomatization on this field suggests that inconsistency ranking may be a more natural concept than inconsistency index, since any monotonic transformation of the latter results in the same ranking. In other words, it makes no sense to differentiate between these variants of a given inconsistency index (see also Brunelli (2016b)).

It is worth to note that our approach is somewhat different from the previous ones in this topic, which aimed at finding a set of suitable properties to be satisfied by any reasonable inconsistency measure. Providing an axiomatic characterization implies neither we accept all properties involved as wholly justified and unquestionable nor we reject the axioms proposed by others. Therefore, the current paper does not argue against the application of other inconsistency indices. The sole implication of our result is that if one agrees with these - rather theoretic - axioms, then the Koczkodaj inconsistency ranking remains the only choice.

Hence we follow characterizations on other fields by assigning modest importance for 
the motivation behind the properties used and for the issue of inconsistency thresholds. Nonetheless, the Koczkodaj index seems to be a reasonable inconsistency index as it meets all reasonable axioms suggested by Brunelli and Fedrizzi (2015); Brunelli (2017); Koczkodaj and Szybowski (2015). In our view, it mitigates the problem caused by the possible hard verification of our properties in practice. Note also that only one index has been associated to a general level of acceptance. However, if a threshold of acceptable inconsistency is given for the Koczkodaj index, then one can find the minimal number of matrix elements to be modified in order to get an acceptable matrix (Bozóki et al., 2015).

The paper is organized as follows. Section 2 presents the main notions and notations. Section 3 introduces the axioms used in the characterization of the Koczkodaj inconsistency ranking offered by Section 4. Independence of the axioms is shown in Section 5. Finally, Section 6 discusses our results and highlights some directions of future research.

\section{Preliminaries}

Let $\mathbb{R}_{+}^{n \times n}$ denote the set of positive matrices (with all elements greater than zero) of size $n \times n$.

Definition 1. Pairwise comparison matrix: Matrix $\mathbf{A}=\left[a_{i j}\right] \in \mathbb{R}_{+}^{n \times n}$ is a pairwise comparison matrix if $a_{j i}=1 / a_{i j}$ for all $1 \leq i, j \leq n$.

In a pairwise comparison matrix, $a_{i j}$ is an assessment of the relative importance of the $i$ th entity with respect to the $j$ th one.

Definition 2. Pairwise comparison submatrix: Let $2 \leq m<n$. Matrix $\mathbf{B}=\left[b_{i j}\right] \in \mathbb{R}_{+}^{m \times m}$ is a pairwise comparison submatrix of pairwise comparison matrix $\mathbf{A}=\left[a_{i j}\right] \in \mathbb{R}_{+}^{n \times n}$ if there exists an injection $\sigma:\{1 ; 2 ; \ldots ; m\} \rightarrow\{1 ; 2 ; \ldots ; n\}$ such that $b_{i j}=a_{\sigma(i) \sigma(j)}$ for all $1 \leq i, j \leq m$. This relation is denoted by $\mathbf{B} \subset \mathbf{A}$.

Definition 3. Triad: Pairwise comparison submatrix $\mathbf{B} \in \mathbb{R}_{+}^{m \times m}$ of pairwise comparison matrix $\mathbf{A} \in \mathbb{R}_{+}^{n \times n}$ is a triad of matrix $\mathbf{A}$ if $m=3$.

The term triad will be analogously used for a pairwise comparison matrix of size $3 \times 3$.

A triad $\mathbf{T}$ can be entirely described by the three elements in its upper triangle: $\mathbf{T}=\left(t_{1} ; t_{2} ; t_{3}\right)$ such that $t_{1}$ is the outcome of the comparison between the 1 st and the 2 nd, $t_{2}$ is the outcome of the comparison between the 1 st and the $3 \mathrm{rd}$, and $t_{3}$ is the outcome of the comparison between the 2 nd and the 3rd entities, respectively.

Definition 4. Consistency: Pairwise comparison matrix $\mathbf{A}=\left[a_{i j}\right] \in \mathbb{R}_{+}^{n \times n}$ is consistent if $a_{i k}=a_{i j} a_{j k}$ for all $1 \leq i, j, k \leq n$.

When the consistency condition does not hold, the pairwise comparison matrix is said to be inconsistent.

Remark 1 . Triad $\mathbf{T}=\left(t_{1} ; t_{2} ; t_{3}\right)$ is consistent if and only if $t_{2}=t_{1} t_{3}$.

Note that a pairwise comparison matrix is consistent if and only if all of its triads are consistent.

The set of all pairwise comparison matrices is denoted by $\mathcal{A}$. Inconsistency is measured by associating a value for each pairwise comparison matrix.

Definition 5. Inconsistency index: Function $I: \mathcal{A} \rightarrow \mathbb{R}$ is an inconsistency index. 
The paper discusses a specific inconsistency indicator, which is the following.

Definition 6. Koczkodaj inconsistency index (Koczkodaj, 1993; Duszak and Koczkodaj, 1994): Consider a pairwise comparison matrix $\mathbf{A}=\left[a_{i j}\right] \in \mathbb{R}_{+}^{n \times n}$. Its inconsistency according to the index $I^{K}$ is

$$
I^{K}(\mathbf{A})=\max _{i<j<k}\left(\min \left\{\left|1-\frac{a_{i k}}{a_{i j} a_{j k}}\right| ;\left|1-\frac{a_{i j} a_{j k}}{a_{i k}}\right|\right\}\right)
$$

Note that the Koczkodaj inconsistency index is based on the maximally inconsistent triad such that its inconsistency is measured by its deviation from a consistent triad.

Inconsistency ranking $\succeq$ is a linear order (complete, antisymmetric and transitive binary relation) on the set of all pairwise comparison matrices $\mathcal{A}$ with respect to inconsistency. $\mathbf{A} \succeq \mathbf{B}$ is interpreted such that pairwise comparison matrix $\mathbf{A}$ is at most as inconsistent as matrix $\mathbf{B}$. The relations $\sim$ and $\succ$ are derived from $\succeq$ in the usual way.

Any inconsistency index induces an inconsistency ranking: $\mathbf{A} \succeq \mathbf{B}$ if and only if matrix A has a not worse (typically, not larger) value of inconsistency than matrix $\mathbf{B}$ according to the given index.

Definition 7. Koczkodaj inconsistency ranking: Consider pairwise comparison matrices $\mathbf{A}=\left[a_{i j}\right] \in \mathbb{R}_{+}^{n \times n}$ and $\mathbf{B}=\left[b_{i j}\right] \in \mathbb{R}_{+}^{m \times m}$. Then $\mathbf{A} \succeq^{K} \mathbf{B}$ if

$$
\max _{i<j<k}\left(\max \left\{\frac{a_{i j} a_{j k}}{a_{i k}} ; \frac{a_{i k}}{a_{i j} a_{j k}}\right\}\right) \leq \max _{i<j<k}\left(\max \left\{\frac{b_{i j} b_{j k}}{b_{i k}} ; \frac{b_{i k}}{b_{i j} b_{j k}}\right\}\right) .
$$

The Koczkodaj inconsistency ranking is the inconsistency ranking induced by the Koczkodaj inconsistency index since, for any triad $\mathbf{T}=\left(t_{1} ; t_{2} ; t_{3}\right)$, there exists a differentiable one-to-one correspondence between the Koczkodaj inconsistency index and the inconsistency index $I^{T}(\mathbf{T})=\max \left\{\left(t_{1} t_{3}\right) / t_{2} ; t_{2} /\left(t_{1} t_{3}\right)\right\}$ (Bozóki and Rapcsák, 2008, Theorem 3.1).

\section{Axioms for an inconsistency ranking}

We start by giving some properties that one could expect an inconsistency ranking to satisfy. After that, they will be commented.

Axiom 1. Positive responsiveness $(P R)$ : Consider two triads $\mathbf{S}=\left(1 ; s_{2} ; 1\right)$ and $\mathbf{T}=$ $\left(1 ; t_{2} ; 1\right)$ such that $s_{2}, t_{2} \geq 1$. Inconsistency ranking $\succeq$ satisfies $P R$ if $\mathbf{S} \succeq \mathbf{T} \Longleftrightarrow s_{2} \leq t_{2}$.

Axiom 2. Invariance under inversion of preferences $(I I P)$ : Consider a triad $\mathbf{T}$ and its transpose $\mathbf{T}^{\top}$. Inconsistency ranking $\succeq$ satisfies $I I P$ if $\mathbf{T} \sim \mathbf{T}^{\top}$.

Axiom 3. Homogeneous treatment of entities (HTE): Consider the triads $\mathbf{T}=\left(1 ; t_{2} ; t_{3}\right)$ and $\mathbf{T}^{\prime}=\left(1 ; t_{2} / t_{3} ; 1\right)$. Inconsistency ranking $\succeq$ satisfies $H T E$ if $\mathbf{T} \sim \mathbf{T}^{\prime}$.

Axiom 4. Scale invariance $(S I)$ : Take the triads $\mathbf{T}=\left(t_{1} ; t_{2} ; t_{3}\right)$ and $\mathbf{T}^{\prime}=\left(k t_{1} ; k^{2} t_{2} ; k t_{3}\right)$. Inconsistency ranking $\succeq$ satisfies $S I$ if $\mathbf{T} \sim \mathbf{T}^{\prime}$ for all $k>0$.

Axiom 5. Monotonicity $(M O N)$ : Consider a pairwise comparison matrix $\mathbf{A}$ and its triad $\mathbf{T}$. Inconsistency ranking $\succeq$ satisfies $M O N$ if $\mathbf{A} \preceq \mathbf{T}$. 
Axiom 6. Reducibility (RED): Consider a pairwise comparison matrix A. Inconsistency ranking $\succeq$ satisfies $R E D$ if $\mathbf{A}$ has a triad $\mathbf{T}$ such that $\mathbf{A} \sim \mathbf{T}$.

Positive responsiveness is based on the observation that triad $\mathbf{T}$ differs more from the basic consistent triad of $(1 ; 1 ; 1)$ than triad $\mathbf{S}$. It is a similar, but more relaxed requirement than monotonicity on single comparisons, proposed by Brunelli and Fedrizzi (2015): PR contains only one implication instead of two, and the condition is demanded for triads, not for all pairwise comparison matrices. ${ }^{2}$

Invariance under inversion of preferences means that inverting all the preferences does not affect inconsistency. This axiom was introduced in Brunelli (2017) for all pairwise comparison matrices. A fundamental reason for this requirement is that $a_{i j}$ is an assessment of the relative importance of the $i$ th entity with respect to the $j$ th one. However, it may equivalently reflect the relative importance of the $j$ th entity with respect to the $i$ th one. The two values are logically reciprocal, but one gets the pairwise comparison matrix $\mathbf{A}^{\top}$ instead of $\mathbf{A}$ (Bozóki and Rapcsák, 2008). Therefore, triads $\mathbf{T}$ and $\mathbf{T}^{\top}$ should be in the same equivalence class of inconsistency.

Homogeneous treatment of entities can be justified in the following way. According to triads $\mathbf{T}$ and $\mathbf{T}^{\prime}$, we have two entities (the 1 st and the 2 nd) equally important on their own. After they are compared with a third entity, the inconsistency of the resulting triad should not depend on the relative importance of the new entity. The violation of HTE would mean that inconsistency is influenced by the absolute importance of entities, contradicting to the notion of pairwise comparisons.

Scale invariance means that the level of inconsistency is independent from the mathematical representation of the preferences. For example, take the $i$ th, the $j$ th and the $k$ th entities such that the $i$ th is 'moderately more important' than $j$ th and $j$ th is 'moderately more important' than the $k$ th with the same intensity of the two relations. Then it makes sense to expect the inconsistency ranking to be the same if 'moderately more important' is identified by the number 2 , or 3 , or 4 , and so on, even allowing for a change in the direction of the two preferences. Naturally, this transformation should preserve consistency, which finalizes the definition of $S I$.

Monotonicity is formulated on the idea that 'no pairwise comparison submatrix may have a worse inconsistency indicator than the given pairwise comparison matrix' by Jacek Szybowski in Koczkodaj and Szybowski (2015). Hence an extension of the set of entities compared cannot improve on inconsistency. For our characterization, it is enough to expect that no triad of the original matrix is allowed to be more inconsistent.

Reducibility ensures that there exists a 'critical' triad whose inconsistency is responsible for the inconsistency of the original pairwise comparison matrix. It will be a crucial axiom in the main result, simplifying the problem by conmtracting its size. Readers familiar with characterizations in other fields of science will not be surprised: for example, the reduced game property is widely used in axiomatizations of different game theory concepts (see e.g. Davis and Maschler (1965)).

Note that $M O N$ and RED together imply monotonicity not only for triads, but for all pairwise comparison submatrices, similarly to the definition of monotonicity by Koczkodaj and Szybowski (2015).

2 The term 'monotonicity' is not used in the name of this property in order to avoid confusion with $M O N$. 


\section{Characterization of the Koczkodaj inconsistency ranking}

Theorem 1. The Koczkodaj inconsistency ranking is the unique inconsistency ranking satisfying positive responsiveness, invariance under inversion of preferences, homogeneous treatment of entities, scale invariance, monotonicity and reducibility.

Proof. We will first argue that the Koczkodaj inconsistency ranking satisfies all axioms. $P R, H T E$ and $S I$ immediately follows from (2). IIP is met due to taking the maximum of $a_{i j} a_{j k} / a_{i k}$ and its inverse in (2). The Koczkodaj inconsistency ranking considers only the maximally inconsistent triad, and the set of triads of a pairwise comparison submatrix is a subset of the set of triads of the original matrix, therefore it meets $M O N$ and $R E D$.

Uniqueness: it is shown that any inconsistency ranking satisfying the six axioms coincides with the Koczkodaj inconsistency ranking.

Assume that there exist pairwise comparison matrices $\mathbf{A}$ and $\mathbf{B}$ such that $\mathbf{A} \succeq \mathbf{B}$ according to an inconsistency ranking $\succeq$, which meets $P R, I I P, H T E, S I, M O N$ and $R E D$. The idea is to gradually simplify - with the use of the axioms - the comparison of inconsistencies of pairwise comparison matrices $\mathbf{A}$ and $\mathbf{B}$ by considering matrices with the same inconsistency level until their ranking depends on a single mathematical relation.

1. Due to $R E D$, there exist triads $\mathbf{A}_{\mathbf{1}}=\left(a_{1} ; a_{2} ; a_{3}\right)$ and $\mathbf{B}_{\mathbf{1}}=\left(b_{1} ; b_{2} ; b_{3}\right)$ such that $\mathbf{A}_{1} \sim \mathbf{A}$ and $\mathbf{B}_{1} \sim \mathbf{B}$.

2. $M O N$ provides that $\mathbf{A}_{\mathbf{1}} \preceq \mathbf{A}^{\prime}$ for all triads $\mathbf{A}^{\prime} \subset \mathbf{A}$ and $\mathbf{B}_{\mathbf{1}} \preceq \mathbf{B}^{\prime}$ for all triads $\mathbf{B}^{\prime} \subset \mathbf{B}$, respectively.

3. Consider $\mathbf{A}_{\mathbf{2}}=\left(1 ; a_{2} / a_{1}^{2} ; a_{3} / a_{1}\right)$ and $\mathbf{B}_{\mathbf{2}}=\left(1 ; b_{2} / b_{1}^{2} ; b_{3} / b_{1}\right) . \quad S I$ implies that $\mathbf{A}_{2} \sim \mathbf{A}_{1}$ and $\mathbf{B}_{2} \sim \mathbf{B}_{1}$.

4. Consider $\mathbf{A}_{\mathbf{3}}=\left(1 ; a_{2} /\left(a_{1} a_{3}\right) ; 1\right)$ and $\mathbf{B}_{\mathbf{3}}=\left(1 ; b_{2} /\left(b_{1} b_{3}\right) ; 1\right)$. HTE results in $\mathbf{A}_{3} \sim \mathbf{A}_{2}$ and $\mathbf{B}_{3} \sim \mathbf{B}_{2}$.

5. IIP provides that $a_{2} /\left(a_{1} a_{3}\right) \geq 1$ and $b_{2} /\left(b_{1} b_{3}\right) \geq 1$ can be assumed without loss of generality in $\mathbf{A}_{\mathbf{3}}$ and $\mathbf{B}_{\mathbf{3}}$, respectively.

6. To summarize, $\mathbf{A}_{3} \sim \mathbf{A}_{2} \sim \mathbf{A}_{1} \sim \mathbf{A} \succeq \mathbf{B} \sim \mathbf{B}_{1} \sim \mathbf{B}_{2} \sim \mathbf{B}_{3}$. It leads to $1 \leq a_{2} /\left(a_{1} a_{3}\right) \leq b_{2} /\left(b_{1} b_{3}\right)$ because of the property $P R$.

However, $1 \leq a_{2} /\left(a_{1} a_{3}\right) \leq b_{2} /\left(b_{1} b_{3}\right)$ means that $\mathbf{A}_{\mathbf{1}} \succeq^{K} \mathbf{B}_{\mathbf{1}}$ due to Definition 6. Analogously, since $\mathbf{A}_{\mathbf{1}} \preceq \mathbf{A}^{\prime}$ for all triads $\mathbf{A}^{\prime} \subset \mathbf{A}$ and $\mathbf{B}_{\mathbf{1}} \preceq \mathbf{B}^{\prime}$ for all triads $\mathbf{B}^{\prime} \subset \mathbf{B}$, we get $\mathbf{A}_{\mathbf{1}} \preceq^{K} \mathbf{A}^{\prime}$ for all triads $\mathbf{A}^{\prime} \subset \mathbf{A}$ and $\mathbf{B}_{\mathbf{1}} \preceq^{K} \mathbf{B}^{\prime}$ for all triads $\mathbf{B}^{\prime} \subset \mathbf{B}$, respectively. It is equivalent to $\mathbf{A} \sim \mathbf{A}_{\mathbf{1}} \succeq{ }^{K} \mathbf{B}_{\mathbf{1}} \sim \mathbf{B}$, so $\succeq$ is the Koczkodaj inconsistency ranking.

The six properties can be classified in at least two ways. The proof of Theorem 1 reveals that the first four axioms (PR,IIP, HTE and SI) characterize an inconsistency ranking on the set of triads, while $M O N$ and $R E D$ are responsible for its extension to pairwise comparison matrices with at least four entities. Therefore, by accepting $M O N$ and $R E D$, other inconsistency rankings based on the maximally inconsistent triad can be characterized. From another perspective, two axioms, $P R$ and $M O N$ contain a 'preference' relation, whereas IIP, HTE and SI define some equivalence classes. RED plays a special role by imposing equivalence between matrices of different size. 
The six axioms above (PR, IIP, HTE, SI, MON and RED) are not enough to uniquely determine the Koczkodaj inconsistency index $I^{K}$ as any monotonic function of it generates the same inconsistency ranking. It means that some technical axioms are necessary in order to obtain the Koczkodaj inconsistency index.

Definition 8. Consistency detection $(C T)$ : Consider a consistent triad T. Inconsistency index $I$ satisfies $C T$ if $I(\mathbf{T})=0$.

$C T$ was suggested by Koczkodaj and Szybowski (2015) and is a specific version of the property Existence of a unique element representing consistency in Brunelli and Fedrizzi (2015). Nonetheless, adding $C T$ to the previous properties is still not enough to exclude some transformations of $I^{K}$. Since it does not make much sense to differentiate between inconsistency indices which generate the same inconsistency ranking (Brunelli, 2016b), the axiomatic characterization of the Koczkodaj inconsistency index remains a topic of future research.

\section{Independence of the axioms}

According to Theorem 1, the six properties determine a unique inconsistency ranking. However, it may turn out that there is some redundancy in the result, certain axioms can be left out. Therefore, six examples, all of them different from the Koczkodaj inconsistency ranking, are given such that they satisfy all but one properties.

Example 1. Consider two pairwise comparison matrices $\mathbf{A}=\left[a_{i j}\right] \in \mathbb{R}_{+}^{n \times n}$ and $\mathbf{B}=$ $\left[b_{i j}\right] \in \mathbb{R}_{+}^{m \times m}$. Then $\mathbf{A} \succeq^{1} \mathbf{B}$ if

$$
\min _{i<j<k}\left(\max \left\{\frac{a_{i j} a_{j k}}{a_{i k}} ; \frac{a_{i k}}{a_{i j} a_{j k}}\right\}\right) \geq \min _{i<j<k}\left(\max \left\{\frac{b_{i j} b_{j k}}{b_{i k}} ; \frac{b_{i k}}{b_{i j} b_{j k}}\right\}\right) .
$$

Lemma 1. The inconsistency ranking $\succeq^{1}$ in Example 1 meets IIP, HTE, SI, MON and RED, but fails PR.

Proof. IIP, HTE and SI contain an equivalence relation on the set of triads, and the inconsistency ranking $\succeq^{1}$ shows the same behaviour as the Koczkodaj inconsistency ranking from this point of view. Inconsistency ranking $\succeq^{1}$ is also based on triads, so it meets $R E D$. Monotonicity is satisfied because minimum over a subset cannot be larger than minimum over the original set. However, it does not satisfy $P R$ since it completely reverses the ranking of triads.

Example 2. Consider two pairwise comparison matrices $\mathbf{A}=\left[a_{i j}\right] \in \mathbb{R}_{+}^{n \times n}$ and $\mathbf{B}=$ $\left[b_{i j}\right] \in \mathbb{R}_{+}^{m \times m}$. Then $\mathbf{A} \succeq^{2} \mathbf{B}$ if

$$
\max _{i<j<k} \frac{a_{i j} a_{j k}}{a_{i k}} \leq \max _{i<j<k} \frac{b_{i j} b_{j k}}{b_{i k}}
$$

Lemma 2. The inconsistency ranking $\succeq^{2}$ in Example 2 meets PR, HTE, SI, MON and RED, but fails IIP.

Proof. PR, HTE and SI consider only the upper triangle of a triad, thus they are satisfied. Inconsistency ranking $\succeq^{2}$ is based on the maximally inconsistent triad, and the set of 
triads of a pairwise comparison submatrix is a subset of the set of triads of the original matrix, therefore it satisfies $M O N$ and $R E D$.

Take the following triads:

$$
\mathbf{A}=\left[\begin{array}{lll}
1 & 1 & 1 \\
1 & 1 & 1 \\
1 & 1 & 1
\end{array}\right] \quad \text { and } \quad \mathbf{B}=\left[\begin{array}{ccc}
1 & 1 & 2 \\
1 & 1 & 1 \\
1 / 2 & 1 & 1
\end{array}\right]
$$

Now $1>1 / 2$ implies $\mathbf{A} \prec^{2} \mathbf{B}$, but $1<2$, so $\mathbf{A}^{\top} \succ^{2} \mathbf{B}^{\top}$, showing the violation of invariance under inversion of preferences.

Example 3. Consider two pairwise comparison matrices $\mathbf{A}=\left[a_{i j}\right] \in \mathbb{R}_{+}^{n \times n}$ and $\mathbf{B}=$ $\left[b_{i j}\right] \in \mathbb{R}_{+}^{m \times m}$. Then $\mathbf{A} \succeq^{3} \mathbf{B}$ if

$$
\max _{i<j<k}\left(\max \left\{\frac{a_{j k}^{2}}{a_{i k}} ; \frac{a_{i k}}{a_{j k}^{2}}\right\}\right) \leq \max _{i<j<k}\left(\max \left\{\frac{b_{j k}^{2}}{b_{i k}} ; \frac{b_{i k}}{b_{j k}^{2}}\right\}\right) .
$$

Lemma 3. The inconsistency ranking $\succeq^{3}$ in Example 3 meets PR, IIP, SI, MON and RED, but fails HTE.

Proof. $P R$ is satisfied because of the analogous role of $a_{i k}$ in (2) and (5). IIP and SI is met due to the substitution of $a_{i j}$ with $a_{j k}$. Inconsistency ranking $\succeq^{3}$ is based on the maximally inconsistent triad, and the set of triads of a pairwise comparison submatrix is a subset of the set of triads of the original matrix, therefore it satisfies $M O N$ and $R E D$.

Take the following triads:

$$
\mathbf{A}=\left[\begin{array}{ccc}
1 & 1 & 3 \\
1 & 1 & 2 \\
1 / 3 & 1 / 2 & 1
\end{array}\right] \quad \text { and } \quad \mathbf{B}=\left[\begin{array}{ccc}
1 & 1 & 5 \\
1 & 1 & 4 \\
1 / 5 & 1 / 4 & 1
\end{array}\right]
$$

Now $2^{2} / 3<4^{2} / 5$ implies $\mathbf{A} \succ^{3} \mathbf{B}$. A possible transformation of triads $\mathbf{A}$ and $\mathbf{B}$ according to $H T E$ leads to $\mathbf{A}^{\prime}=(1 ; 3 / 2 ; 1)$ and $\mathbf{B}^{\prime}=(1 ; 5 / 4 ; 1)$, so $\mathbf{A}^{\prime} \prec^{3} \mathbf{B}^{\prime}$ since $3 / 2>5 / 4$. It reveals the violation of homogeneous treatment of entities by the inconsistency ranking $\succeq^{3}$.

Example 4. Consider two pairwise comparison matrices $\mathbf{A}=\left[a_{i j}\right] \in \mathbb{R}_{+}^{n \times n}$ and $\mathbf{B}=$ $\left[b_{i j}\right] \in \mathbb{R}_{+}^{m \times m}$. Then $\mathbf{A} \succeq^{4} \mathbf{B}$ if

$$
\max _{i<j<k}\left(\max \left\{\frac{a_{j k}}{a_{i k}} ; \frac{a_{i k}}{a_{j k}}\right\}\right) \leq \max _{i<j<k}\left(\max \left\{\frac{b_{j k}}{b_{i k}} ; \frac{b_{i k}}{b_{j k}}\right\}\right) .
$$

Lemma 4. The inconsistency ranking $\succeq^{4}$ in Example 4 meets PR, IIP, HTE, MON and RED, but fails $S I$.

Proof. PR is satisfied because of the analogous role of $a_{i k}$ in (2) and (6). IIP and HTE hold as only $a_{i j}$ is eliminated from the original definition. Inconsistency ranking $\succeq^{4}$ is based on the maximally inconsistent triad, and the set of triads of a pairwise comparison submatrix is a subset of the set of triads of the original matrix, therefore it satisfies $M O N$ and $R E D$.

Take the following triads:

$$
\mathbf{A}=\left[\begin{array}{lll}
1 & 1 & 1 \\
1 & 1 & 1 \\
1 & 1 & 1
\end{array}\right] \quad \text { and } \quad \mathbf{A}^{\prime}=\left[\begin{array}{ccc}
1 & 2 & 4 \\
1 / 2 & 1 & 2 \\
1 / 4 & 1 / 2 & 1
\end{array}\right]
$$


Now $1 / 1>2 / 4$ implies $\mathbf{A} \prec^{4} \mathbf{A}^{\prime}$, but $\mathbf{A}^{\prime}$ can be obtained by a transformation of triad $\mathbf{A}$ according to $S I$, showing the violation of scale invariance.

Example 5. Consider two pairwise comparison matrices $\mathbf{A}=\left[a_{i j}\right] \in \mathbb{R}_{+}^{n \times n}$ and $\mathbf{B}=$ $\left[b_{i j}\right] \in \mathbb{R}_{+}^{m \times m}$. Then $\mathbf{A} \succeq^{5} \mathbf{B}$ if

$$
\min _{i<j<k}\left(\max \left\{\frac{a_{i j} a_{j k}}{a_{i k}} ; \frac{a_{i k}}{a_{i j} a_{j k}}\right\}\right) \leq \min _{i<j<k}\left(\max \left\{\frac{b_{i j} b_{j k}}{b_{i k}} ; \frac{b_{i k}}{b_{i j} b_{j k}}\right\}\right) .
$$

Lemma 5. The inconsistency ranking $\succeq^{5}$ in Example 5 meets PR, IIP, HTE, SI and RED, but fails $M O N$.

Proof. Inconsistency ranking $\succeq^{5}$ is equivalent to the Koczkodaj inconsistency ranking on the set of triads. PR, IIP, HTE and SI consider only a triad, hence these properties are satisfied. There exists a minimally inconsistent triad in any pairwise comparison matrix, so $R E D$ is met. The set of triads of a pairwise comparison submatrix is a subset of the set of triads of the original matrix, therefore inconsistency ranking $\succeq^{5}$ violates $M O N$.

Example 6. Consider two pairwise comparison matrices $\mathbf{A}=\left[a_{i j}\right] \in \mathbb{R}_{+}^{n \times n}$ and $\mathbf{B}=$ $\left[b_{i j}\right] \in \mathbb{R}_{+}^{m \times m}$. Then $\mathbf{A} \succeq^{6} \mathbf{B}$ if

$$
\max _{i<j<k}\left(\max \left\{\frac{a_{i j} a_{j k}}{a_{i k}} ; \frac{a_{i k}}{a_{i j} a_{j k}}\right\}\right)^{n} \leq \max _{i<j<k}\left(\max \left\{\frac{b_{i j} b_{j k}}{b_{i k}} ; \frac{b_{i k}}{b_{i j} b_{j k}}\right\}\right)^{m} .
$$

Lemma 6. The inconsistency ranking $\succeq^{6}$ in Example 6 meets PR, IIP, HTE, SI and $M O N$, but fails RED.

Proof. Inconsistency ranking $\succeq^{6}$ is equivalent to the Koczkodaj inconsistency ranking when $n=m$, for example, on the set of triads. Thus PR, IIP, HTE and SI are all satisfied. Since $\max \left\{a_{i j} a_{j k} / a_{i k} ; a_{i k} /\left(a_{i j} a_{j k}\right)\right\} \geq 1$, inconsistency ranking $\succeq^{6}$ meets $M O N$. However, it violates $R E D$ because of the appearance of $n$ and $m$ in (8).

Table 1 summarizes the above discussion on the independence of the axioms.

Table 1: Axiomatic properties of inconsistency rankings

\begin{tabular}{llllllll}
\hline $\begin{array}{l}\text { Inconsistency } \\
\text { ranking }\end{array}$ & Definition & $P R$ & IIP & $H T A$ & $S I$ & MON & $R E D$ \\
\hline Koczkodaj & Definition 7 & $\checkmark$ & $\checkmark$ & $\checkmark$ & $\checkmark$ & $\checkmark$ & $\checkmark$ \\
\hline$\succeq^{1}$ & Example 1 & $x$ & $\checkmark$ & $\checkmark$ & $\checkmark$ & $\checkmark$ & $\checkmark$ \\
$\succeq^{2}$ & Example 2 & $\checkmark$ & $x$ & $\checkmark$ & $\checkmark$ & $\checkmark$ & $\checkmark$ \\
$\succeq^{3}$ & Example 3 & $\checkmark$ & $\checkmark$ & $x$ & $\checkmark$ & $\checkmark$ & $\checkmark$ \\
$\succeq^{4}$ & Example 4 & $\checkmark$ & $\checkmark$ & $\checkmark$ & $x$ & $\checkmark$ & $\checkmark$ \\
$\succeq^{5}$ & Example 5 & $\checkmark$ & $\checkmark$ & $\checkmark$ & $\checkmark$ & $x$ & $\checkmark$ \\
$\succeq^{6}$ & Example 6 & $\checkmark$ & $\checkmark$ & $\checkmark$ & $\checkmark$ & $\checkmark$ & $x$ \\
\hline
\end{tabular}

Note that inconsistency rankings $\succeq^{1}$ and $\succeq^{5}$ differ only in the direction of the inequality. Analogously to Theorem $1, \succeq^{1}$ is characterized by IIP, HTE, SI, MON, RED (the five axioms it satisfies) and negative responsiveness, the inverse of positive responsiveness defined as $\mathbf{S} \succeq \mathbf{T}$ if and only if $t_{2} \geq s_{2}$. In a similar way, $\succeq^{5}$ is the unique inconsistency 
ranking satisfying $P R, I I P, H T E, S I, R E D$ and reversed monotonicity, that is, $\mathbf{A} \succeq \mathbf{B}$ for any pairwise comparison matrix $\mathbf{A}$ and its triad $\mathbf{B}$.

Inconsistency rankings $\succeq^{1}-\succeq^{5}$ have not much practical use. On the other hand, inconsistency ranking $\succeq^{6}$ may be meaningful if inconsistency is assumed to (deterministically) increase with size due to the possible presence of other inconsistent triads.

\section{Discussion}

The paper has provided an axiomatic characterization of the inconsistency ranking induced by the Koczkodaj inconsistency index. The theorem requires six properties, some of them directly borrowed from previous axiomatic discussions (IIP from Brunelli (2017) and $M O N$ from Koczkodaj and Szybowski (2015)), some of them similar to the axioms used in the literature $(P R$ is a relaxed version of monotonicity on single comparisons by Brunelli and Fedrizzi (2015) and RED is implicitly contained in monotonicity by Koczkodaj and Szybowski (2015)), and some of them introduced here (HTE and SI). Although we have given a few arguments for homogeneous treatment of entities and scale invariance, their justification can be debated. However, it does not influence the essence of our characterization, which only states that the Koczkodaj inconsistency ranking should be accepted when all properties are adopted.

It remains to be seen how this characterization can be modified. The proof of Theorem 1 shows that $I I P$ can be eliminated if $P R$ is defined without the condition $s_{2}, t_{2} \geq 1$. Although it seems to be a simplification (we will have only five axioms instead of six), the current form better reflects the role of different properties, highlighted by the discussion of logical independence in Section 5 .

Another direction can be the use of other known properties in the characterization. A natural candidate is the following one, suggested by Brunelli and Fedrizzi (2015) and applied by Koczkodaj and Szybowski (2015), too.

Definition 9. Order invariance $(O I)$ : Consider a pairwise comparison matrix $\mathbf{A}$ and a permutation matrix $\mathbf{P}$ of the same size. Inconsistency ranking $\succeq$ satisfies $O I$ if $\mathbf{P A P}^{\top} \sim \mathbf{A}$.

$O I$ says that changing the order of the entities does not affect the inconsistency of preferences. In our characterization, homogeneous treatment of entities and scale invariance have a somewhat analogous role, but they cannot be substituted immediately by $O I$.

To summarize, the current study clearly does not mean the end of the discussion on inconsistency indices. The main aim of this topic is perhaps the identification of the best universal measure, or at least, the perfect in a certain field. For this purpose, a thorough understanding of inconsistency indicators is indispensable. We hope the current study may give new insights by presenting the first axiomatic characterization in this field for the ranking derived from the Koczkodaj inconsistency index.

\section{Acknowledgements}

I would like to thank to Matteo Brunelli, Michele Fedrizzi, Waldemar W. Koczkodaj and Jacek Szybowski for inspiration. I am also grateful to Matteo Brunelli, Sándor Bozóki and Miklós Pintér for reading the manuscript and for useful advices.

Three anonymous reviewers provided valuable comments and suggestions on earlier drafts. The research was supported by OTKA grant K 111797 and by the MTA Premium Post 
Doctorate Research Program.

This research was partially supported by Pallas Athene Domus Scientiae Foundation. The views expressed are those of the author's and do not necessarily reflect the official opinion of Pallas Athene Domus Scientiae Foundation.

\section{References}

Aguaron, J. and Moreno-Jiménez, J. M. (2003). The geometric consistency index: approximated thresholds. European Journal of Operational Research, 147(1):137-145.

Barzilai, J. (1997). Deriving weights from pairwise comparison matrices. Journal of the Operational Research Society, 48(12):1226-1232.

Barzilai, J. (1998). Consistency measures for pairwise comparison matrices. Journal of Multi-Criteria Decision Analysis, 7(3):123-132.

Bouyssou, D. and Marchant, T. (2014). An axiomatic approach to bibliometric rankings and indices. Journal of Informetrics, 8(3):449-477.

Bozóki, S., Csató, L., and Temesi, J. (2016). An application of incomplete pairwise comparison matrices for ranking top tennis players. European Journal of Operational Research, 248(1):211-218.

Bozóki, S., Fülöp, J., and Poesz, A. (2015). On reducing inconsistency of pairwise comparison matrices below an acceptance threshold. Central European Journal of Operations Research, 23(4):849-866.

Bozóki, S. and Rapcsák, T. (2008). On Saaty's and Koczkodaj's inconsistencies of pairwise comparison matrices. Journal of Global Optimization, 42(2):157-175.

Brunelli, M. (2016a). Recent advances on inconsistency indices for pairwise comparisons a commentary. Fundamenta Informaticae, 144(3-4):321-332.

Brunelli, M. (2016b). A technical note on two inconsistency indices for preference relations: a case of functional relation. Information Sciences, 357:1-5.

Brunelli, M. (2017). Studying a set of properties of inconsistency indices for pairwise comparisons. Annals of Operations Research, 248(1):143-161.

Brunelli, M., Canal, L., and Fedrizzi, M. (2013). Inconsistency indices for pairwise comparison matrices: a numerical study. Annals of Operations Research, 211(1):493509.

Brunelli, M. and Fedrizzi, M. (2011). Characterizing properties for inconsistency indices in the AHP. In Proceedings of the International Symposium on the Analytic Hierarchy Process (ISAHP), pages 15-18.

Brunelli, M. and Fedrizzi, M. (2015). Axiomatic properties of inconsistency indices for pairwise comparisons. Journal of the Operational Research Society, 66(1):1-15. 
Cavallo, B. and D'Apuzzo, L. (2012). Investigating properties of the $\odot$-consistency index. In Greco, S., Bouchon-Meunier, B., Coletti, G., Fedrizzi, M., Matarazzo, B., and Yager, R. R., editors, Advances in Computational Intelligence: 14th International Conference on Information Processing and Management of Uncertainty in KnowledgeBased Systems, IPMU 2012, Catania, Italy, July 9-13, 2012, Proceedings, Part IV, volume 300 of Communications in Computer and Information Science, pages 315-327. Springer, Berlin-Heidelberg.

Davis, M. and Maschler, M. (1965). The kernel of a cooperative game. Naval Research Logistics Quarterly, 12(3):223-259.

Dubey, P. (1975). On the uniqueness of the Shapley value. International Journal of Game Theory, 4(3):131-139.

Duszak, Z. and Koczkodaj, W. W. (1994). Generalization of a new definition of consistency for pairwise comparisons. Information Processing Letters, 52(5):273-276.

Fichtner, J. (1984). Some thoughts about the mathematics of the Analytic Hierarchy Process. Technical report, Institut für Angewandte Systemforschung und Operations Research, Universität der Bundeswehr München.

Fichtner, J. (1986). On deriving priority vectors from matrices of pairwise comparisons. Socio-Economic Planning Sciences, 20(6):341-345.

Hart, S. and Mas-Colell, A. (1989). Potential, value, and consistency. Econometrica, $9(11): 589-614$.

Kendall, M. G. and Smith, B. B. (1940). On the method of paired comparisons. Biometrika, 31(3/4):324-345.

Koczkodaj, W. W. (1993). A new definition of consistency of pairwise comparisons. Mathematical and Computer Modelling, 18(7):79-84.

Koczkodaj, W. W. and Szwarc, R. (2014). On axiomatization of inconsistency indicators for pairwise comparisons. Fundamenta Informaticae, 132(4):485-500.

Koczkodaj, W. W. and Szybowski, J. (2015). Axiomatization of inconsistency indicators for pairwise comparisons matrices revisited. Manuscript. arXiv:1509.03781v1.

Miroiu, A. (2013). Axiomatizing the Hirsch index: Quantity and quality disjoined. Journal of Informetrics, 7(1):10-15.

Peláez, J. I. and Lamata, M. T. (2003). A new measure of consistency for positive reciprocal matrices. Computers $\&$ Mathematics with Applications, 46(12):1839-1845.

Quesada, A. (2010). More axiomatics for the Hirsch index. Scientometrics, 82(2):413-418.

Saaty, T. L. (1977). A scaling method for priorities in hierarchical structures. Journal of Mathematical Psychology, 15(3):234-281.

Saaty, T. L. (1980). The Analytic Hierarchy Process: planning, priority setting, resource allocation. McGraw-Hill, New York. 
Shapley, L. S. (1953). A value for $n$-person games. In Kuhn, H. W. and Tucker, A. W., editors, Contributions to the Theory of Games Volume II, volume 28 of Annals of Mathematical Studies, pages 307-317. Princeton University Press, Princeton, New Jersey.

Stein, W. E. and Mizzi, P. J. (2007). The harmonic consistency index for the analytic hierarchy process. European Journal of Operational Research, 177(1):488-497.

van den Brink, R. (2002). An axiomatization of the Shapley value using a fairness property. International Journal of Game Theory, 30(3):309-319.

Woeginger, G. J. (2008). An axiomatic characterization of the Hirsch-index. Mathematical Social Sciences, 56(2):224-232.

Young, H. P. (1985). Monotonic solutions of cooperative games. International Journal of Game Theory, 14(2):65-72. 